\title{
Development of Purification Process Using Electrocoagulation Technique for Biodiesel Produced via Homogeneous Catalyzed Transesterification Process of Refined Palm Oil
}

\author{
Rossarin Ampairojanawong ${ }^{1}$, Ajalaya Boripun ${ }^{1}$, Sayan Ruankon ${ }^{2}$, Thanapong Suwanasri², and Tawiwan Kangsadan ${ }^{1, *}$ \\ ${ }^{1}$ Chemical and Process Engineering Program, The Sirindhorn International Thai-German Graduate School of Engineering (TGGS), \\ King Mongkut's University of Technology North Bangkok, Bangkok, Thailand \\ ${ }^{2}$ Electrical Power and Energy Engineering Program, The Sirindhorn International Thai-German Graduate School of Engineering \\ (TGGS), King Mongkut's University of Technology North Bangkok, Bangkok, Thailand
}

\begin{abstract}
This work is focused on the preliminary development of purification process using electrocoagulation technique for biodiesel produced via homogeneous catalyzed transesterification process of refined palm oil (RPO) to shorten the separation process and reduce the water consumption during the final purification with water-washing process. Biodiesel as Fatty acid methyl esters (FAMEs) were produced via transesterification reaction of RPO as feedstock with methanol in the presence of sodium hydroxide derivative-catalyst at the optimal condition using conventional heating. Electrocoagulation separation process was in-house designed to investigate the effects of electrode configurations such as shapes, distances between electrodes and suitable applied AC high voltages (ranging from 1 to $9 \mathrm{kV}$ ) on the separation efficiency of glycerol and biodiesel. Results revealed that with the electrocoagulation process at room temperature using $\mathrm{AC}$ electrical current at high voltage of $3 \mathrm{kV}$ (and low amperage) with a point to point electrode configuration and the vertical-distance between electrodes of $3 \mathrm{~cm}$ was very efficient and achieved higher free glycerol removal than using the conventional separation of the gravitational settling. Furthermore, the separation time was significantly shortened from typically $>24 \mathrm{~h}$ with the gravitational settling to $240 \mathrm{~s}(4 \mathrm{~min})$ and the number of water-washing was reduced from 5 to 3 times during the final purification process. The final purity of biodiesel was $98.89 \% \pm 0.25 \%$ with high quality according to standards of EN 14214 and ASTM D6751.
\end{abstract}

\section{Introduction}

Biodiesel is proven to be an alternative diesel fuel with its overall properties, performance and chemical structure are similar to petroleum diesel. Importantly, it is naturally non-toxic and biodegradable with less greenhouse gas emission. Therefore, it can be used as totally replacement or partially blending with petroleum diesel fuel.

Commercially, biodiesel is produced via transesterification of triglycerides such as vegetable oils (e.g. palm oil, soybean, coconut oil, rapeseed oil), animal fats and/or waste cooking oils with alcohol and homogenous alkali-catalysts at low temperature and pressure and the conversion yield is over 98\% [1-3]. A successful transesterification reaction is signified by the separation of biodiesel from by-product of glycerol after the optimized reaction time of $30 \mathrm{~min}$ to $1 \mathrm{~h}[1,2]$. With the density difference and low solubility of glycerol in biodiesel, the separation can be accomplished by gravitational settling. However, excess amounts of unreacted reactants such as alcohol, base catalyst, saturated or unsaturated of glycerides and glycerol are remained in the biodiesel phase and are not easily removed because of its reversible reaction. Moreover, these impurities are dispersed in the biodiesel phase with complex structure. Thus, it is necessary to purify the biodiesel product because these impurities can lead to problems in the operation of engine. Especially, glycerol can shorten the storage life-time of biodiesel.

Common practice for purifying biodiesel is washing with warm water to remove impurities. Nevertheless, water washing has its drawbacks since it requires many hours to complete the separation of biodiesel from water and the large amount of water is consumed and required to be treated later. Additionally, water residual in biodiesel must be efficiently removed by evaporating at high temperature which contributes to relatively high operation costs. Particularly using homogeneous alkalicatalyst, such as sodium hydroxide, the presence of soap can produce emulsified mixture and result in substantial product loss through hydrolysis and saponification reactions. Moreover, the saponification side-reaction can also cause phase separation problems and formation of emulsion during the purification process.

Although, the traditional separation technologies used in biodiesel production includes the gravitational settling $[3,4]$ and the centrifugal force $[5,6]$, but it is inefficient for glycerol and water separation and delays in the purification process while increasing cost of post-

\footnotetext{
Corresponding author: tawiwan.k@tggs.kmutnb.ac.th
} 
treatment. Especially, the centrifuge technique requires high energy with large-volume of sample for rapid separation, resulting in considerably high operation cost, still traces of water and soap are not completely removed. Separation time of biodiesel is ranging from 8 to $24 \mathrm{~h}$ to achieve biodiesel product within the standards $[7,8]$. Therefore, alternative technologies with low energy consumption, low operating cost and high separation efficiency for liquid/liquid separation of glycerol and water from the biodiesel need to be explored. These are decantation [9] and sedimentation [10], filtration or membrane [11] and distillation [12].

Recently, several alternative separation processes and purification procedures with waterless have been developed, such as electrocoagulation or electrostatic separation. Electrocoagulation (EC) technologies are considered reliable and effective process for phase separation in compared with membrane technology which is slow and using expensive chemical reagents or solid absorbents [13]. EC is widely used in various applications such as separation of multi-components, separation of emulsion solvents and treatment wastewater. EC technique can efficiently remove small charge-colloidal particles from neutral-aqueous media by applying electric current causing the aggregation of tiny destabilized particles to larger formation with higher density to be settled under gravitational force. $\mathrm{Al}$ and $\mathrm{Fe}$ electrodes are commonly used [14], however, the removal efficiency of electrocoagulation using $\mathrm{Al}$ electrodes was higher than that of using $\mathrm{Fe}$ electrodes [15].

EC consists of three consecutive principles [16]:

(i) Formation of coagulants by electrolytic oxidation of the sacrificial anode electrodes.

(ii) Destabilization of contaminants, particulate suspension and emulsion breaking.

(iii) Aggregation of destabilized phases to form flocs occurs.

For the destabilization mechanisms, the following phenomena has been observed:

(i) Double layer compression

(ii) Adsorption and charge neutralization

(iii) Agglomeration in a precipitate

(iv) Adsorption and inter-particle bridging

EC processes with a low voltage and high current of direct electrical current (DC) voltage supply were intensively implemented [18] using the concept of electrochemical in which the electric field is generated, and ions are transported because of sacrificial electrodes in the mixture solution. However, the formation of oxide film during electrolysis can lead to metal passivation which decreased the ionic transport and separation efficiency. Alternating electrical current (AC) is more suitable for electrocoagulation process because its cyclic energization where current periodically changes or its flow direction is reversed can prevent the passivation of electrode or reduce the corrosion of anode and cathode electrode at low energy consumption [19]. In comparing with DC voltage converter, the set-up of AC voltage system is simpler by using a transformer to change the voltage, less complicated, more reliable, easy to operation, no chemical additions, required small spacing and shortened reactive retention time (rapid sediments).

This work aims to implement EC technology with $\mathrm{AC}$ high voltage ranging from 1 to $9 \mathrm{kV}$ system for the separation of biodiesel and glycerol with shortest separation time while eliminating other un-reacted reactants, catalyst and soap to achieve high quality biodiesel within the standards. Consequently, water consumption during the purification process can be reduced. This EC separation process with high voltage could complement to previously developed single-mode microwave-assisted reactor for biodiesel production using homogeneous catalyzed transesterification reaction by our research group [20-22] in which this reactor can enhance the reaction within $400 \mathrm{~s}$. Therefore, it would be more practical for the commercialization once the separation can be accomplished within the same order of time. Moreover, this EC technology was also applied for the purification of crude glycerol which obtained after the separation of biodiesel and glycerol step [23].

\section{Materials and methods}

\subsection{Materials}

Fatty acids composition of refined palm oil (RPO) used as a raw material were shown in Table 1. Chemical reagents were methanol with $99.9 \%$ purity, sodium hydroxide derivative-catalyst and n-heptane manufactured by RCI Labscan Limited. All standard chemicals used for the biodiesel analysis according to EN 14103 [24] were supplied by the Sigma-Aldrich Chemicals Company. GC-FID analysis also indicated that RPO fraction of monoacylglycerols or monoglyceride (MG) and Diacylglycerols or Diglyceride (DG) were $0.08 \pm 0.05 \%$ and $6.22 \pm 0.65 \%$, respectively.

Table 1. Fatty acids composition of refined palm oil (RPO) obtained from gas chromatography analysis.

\begin{tabular}{|c|c|c|c|}
\hline Type & Name & Formula & Composition (\%w/w) \\
\hline \multirow{5}{*}{ Saturated } & Luaric acid & $\mathrm{C} 12: 0$ & 0.80 \\
\cline { 2 - 4 } & Myristic acid & $\mathrm{C} 14: 0$ & 1.10 \\
\cline { 2 - 4 } & Palmitic acid & $\mathrm{C} 16: 0$ & 43.45 \\
\cline { 2 - 4 } & Stearic acid & $\mathrm{C} 18: 0$ & 4.23 \\
\cline { 2 - 4 } & Arachidic acid & $\mathrm{C} 20: 0$ & 0.16 \\
\cline { 2 - 4 } & Behenic acid & $\mathrm{C} 22: 0$ & 0.06 \\
\cline { 2 - 4 } & Erucic acid & $\mathrm{C} 24$ isomer & 0.07 \\
\hline \multirow{4}{*}{ Unsaturated } & Oleic acid & $\mathrm{C} 18: 1$ & 40.90 \\
\cline { 2 - 4 } & Linoleic acid & $\mathrm{C} 18: 2$ & 9.10 \\
\cline { 2 - 4 } & Linoleic acid & $\mathrm{C} 18: 3$ & 0.24 \\
\hline
\end{tabular}




\subsection{Methods}

Experimental procedures were carried out into 3 parts: (1) the production of biodiesel and its by-product glycerol; (2) the separation of glycerol from biodiesel; and (3) the analysis of final products.

\subsubsection{Transesterification reaction process}

Biodiesel and by-product glycerol were produced via homogeneous catalyzed transesterification reaction using conventional heating as shown in Fig. 1. Refined palm oil (RPO) was preheated in a thermal bath until the temperature reached to $60^{\circ} \mathrm{C}$ before adding the methanol and catalyst solution. The reaction operated at the optimum condition which obtained from previous studies [20-22]. Fig. 1 shows the schematic diagram of the labscale biodiesel production setup. The reaction of optimum condition was performed in three-neck flask reactor with a constant temperature control in water bath and agitated vigorously with a mechanical overhead stirrer. To ensure the complete reaction, the reaction time was proceeded for $1 \mathrm{~h}$. At the end of reaction, the product mixture containing biodiesel, glycerol, methanol, remaining catalyst and unreacted reactants was first separated using the gravitational settling (GS) by pouring into a separating funnel and allowed to settle until the clear interface between biodiesel and glycerol layers were observed. This would ensure that glycerol was completely separated from the biodiesel and obtained two layers comprising an upper layer as biodiesel and a lower layer as glycerol, catalyst and unreacted mixture. These two products were collected separately without water-washing.

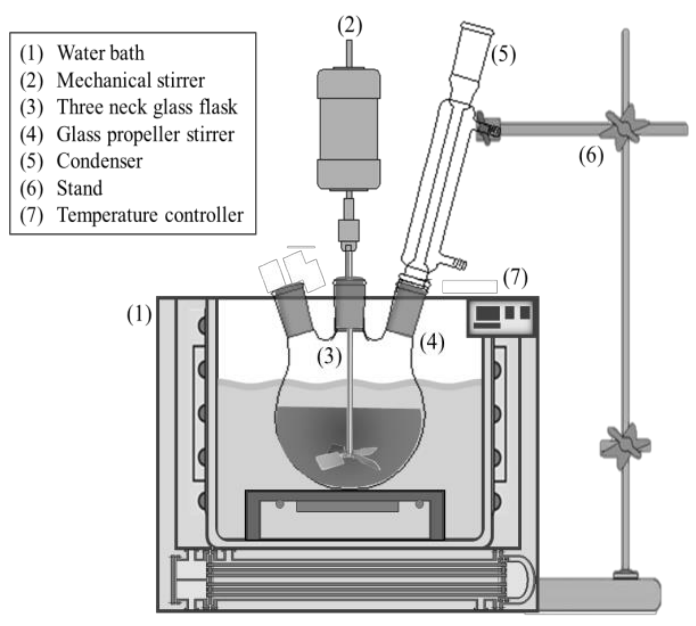

Fig. 1. Schematic diagram of biodiesel production via homogeneous catalyzed transesterification reaction using conventional heating in the water bath.

\subsubsection{Electrocoagulation separation process}

In this study, to separate glycerol from glycerolbiodiesel mixture, an electrostatic field caused by a high voltage (more than $\mathrm{kV}$ ) and low amperage $\mathrm{AC}$ current $(\mathrm{mA})$ was used. The separation technique was tested on refined palm oil-based produced biodiesel. All experiments were performed in electrocoagulation cylinder setup and the volume of the solution of each run for electrocoagulation separation was $1200 \mathrm{~mL}$. The unit consisted of one pair of Fe electrode were connected to the power source and the diameter of each electrode was $3.2 \mathrm{~mm}$. AC power supply with an input of $220 \mathrm{~V}$ and variable output of $1,3,5,7$ and $9 \mathrm{kV}$ with low current of ampere was used as direct current source with run reaction of EC separation at room temperature. The instrument setup (Fig. 2) mainly consisted of auto transformer, step-up transformer, AC voltage divider, $\mathrm{AC}$ volt meter, $\mathrm{AC}$ amp meter, cable connector and resistor. High-voltage tests performed in faraday cage to ensure the safety and some of power equipment and the insulation of these devices must be well designed to sustain high electrical stress.

The biodiesel and glycerol product mixture collected in the previous step with the total volume of $1200 \mathrm{~mL}$ were mixed in the cylindrical chamber (diameter of 8.5 $\mathrm{cm}$ and height of $30 \mathrm{~cm}$ with the maximum capacity of $1700 \mathrm{~mL}$ ) and vigorously stirred for $30 \mathrm{~min}$ and maintained the temperature at $60^{\circ} \mathrm{C}$ (same discharge temperature from the transesterification reactor). Then, the electrocoagulation process was immediately started by immersing anode and cathode electrodes into the product mixture at the center of cylindrical chamber and then connecting the electrical circuit as shown in Fig. 2.

Anode and cathode electrodes were made from iron $(\mathrm{Fe})$ in a rod with $3.2 \mathrm{~mm}$ in diameter and the tips of electrode were in bar and point shapes as shown in Fig. 3. Five different electrode configurations were investigated by varying distances between two electrodes and types of electrode. The horizontal distance between inter-electrodes A and B were at a constant of $3.5 \mathrm{~cm}$.

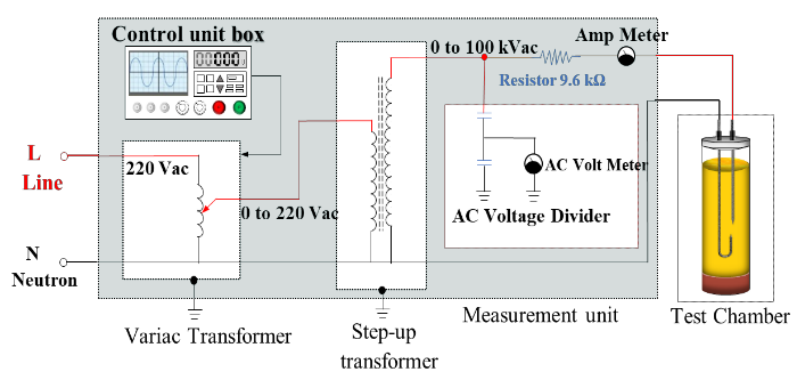

Fig. 2. Schematic diagram of glycerol separation from biodiesel using electrocoagulation process (EC) with AC electrical current at high voltage. 


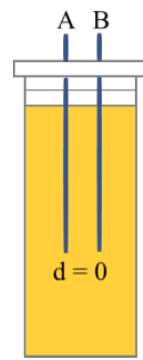

(a)

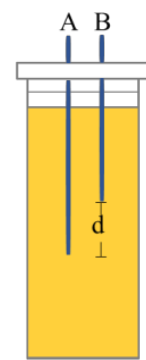

(b)

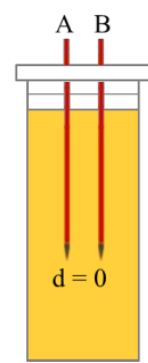

(c)

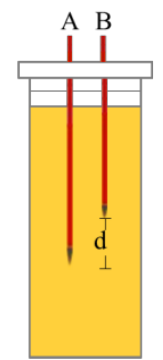

(d)

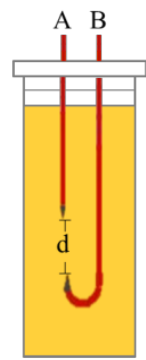

(e)

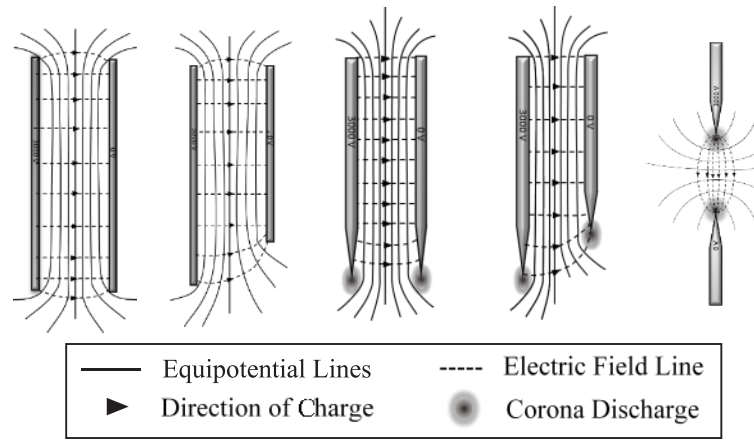

Fig. 3. Configuration of electrodes (type and arrangement): (a) bar electrodes at the same level $(\mathrm{d}=0)$, (b) bar electrodes at different level with electrode distance (d), (c) point electrodes at the same level $(\mathrm{d}=0),(\mathrm{d})$ point electrodes at different level with electrode distance (d), and (e) point-to-point electrodes with electrode distance (d).

Electric fields were represented by drawing field lines that indicated the direction of the field, as well as the strength of the field as shown in Fig. 3. More field lines represented a higher field strength. Non-uniform electric field showed that the field lines tended to be curved and were more concentrated near the charges. With a uniform electric field, the field strength did not vary, therefore, the field lines were parallel to each other and equally spaced. Uniform fields were created by setting up a potential difference between two conducting bars placed at a certain distance from one another. The field was considered to be uniform at the center of the bars but varies close to the edge of the bars as showed in Fig. 3(a) and (b).

The strength of the electrical field depends on the potential difference applied to two electrodes and the distance at which they are separated. A higher potential difference or voltage results in a stronger electric field. The greater the distance between electrodes, the weaker the field becomes. The non- uniform electric distribution creates electrical stress within the surface of dielectric barrier. As the distance between the electrodes increased, the electric field decreases. Therefore, it is necessary to study the distance between two electrodes to ensure the generation of electrical field and to prevent the electrical shock which can lead to the explosion.

By applying high voltage between bar or point electrode, charges are accumulated in area of tip electrodes in which increased the electrical stress and then ions in liquid phase are ionized as free charges and the conductivity of liquid increase. In this work, the high voltage in the range of $\mathrm{kV}$ was applied to the medium, this allowed temporarily dipole induction of nonpolar molecules [25]. Note that glycerol has the average dielectric constant of 42.5 [26] which is much higher than the biodiesel (average dielectric constant of 3.245 $[27,28])$. Besides two electrodes, this area of discharge can increase the conductivity and allow the exchange between charges of glycerol and other charge molecules such as remaining catalysts and soap resulting in larger agglomerations and then precipitating to the bottom under the influence of gravitational force. In this work studies, two shapes of electrode tips were investigated: bar (with flat cross-sectional area) and point (with sharp cross-sectional area). The corresponding electrical fields for different shapes of tips and configurations were illustrated in Fig. 3.

After applying AC electrical current at desired high voltage, the product mixture was separated until observing the clear interface between biodiesel and glycerol layers. Separation efficiency was determined, and the purity of biodiesel, remaining catalyst and soap contents were analyzed

\subsubsection{Purification process}

After the completion of glycerol and contaminants separation using EC, the crude biodiesel was purified by washing with warm water. The process was repeated until colorless washed water was obtained. Then remaining methanol and water were eliminated from biodiesel with a rotary evaporator by applying a vacuum of 100 mbar at $60^{\circ} \mathrm{C}$ and $90^{\circ} \mathrm{C}$, respectively, in order to ensure that the high quality of biodiesel product met the standards (EN 14214 or ASTM D 6751 and EN 14105).

\subsection{Analysis methods}

The separated biodiesel and glycerol product samples were analyzed for EC separation efficiency based on the removal of glycerol and then later compared the performance with the gravitational settling (GS) separation method. Furthermore, the biodiesel product was analyzed for its purity as methyl ester content (\%) using Gas Chromatography (GC) according to EN14103 standard as well as remaining catalyst and soap contents in normalized values.

\subsubsection{Separation efficiency}

The performance of individual EC separation conditions was compared by measuring removal glycerol from biodiesel. Then, the separation efficiency $(\% \mathrm{w} / \mathrm{w})$ was calculated using Eq. (1).

$$
\eta_{g}(\%)=\left(\frac{V_{f}}{V_{i}}\right) \times 100 \%
$$

where $V_{i}$ is the initial volume of glycerol and $V_{f}$ is the final volume of glycerol after the separation. 


\subsubsection{Fatty acid methyl ester content}

Fatty Acid Methyl Ester (FAME) content was analyzed according to the standard method EN 14103 [22] using gas chromatography (Shimadzu GC-2010) with Flame Ionization Detector (FID) by the capillary column used as DB-WAX column with length of $30 \mathrm{~m}$, internal diameter $0.250 \mathrm{~mm}$ and coated with thickness film 0.25 $\mu \mathrm{m}$. Nitrogen was used as carrier gas as well as detector make up gas with constant flow rate $1 \mathrm{~mL} / \mathrm{min}$ and 30 $\mathrm{mL} / \mathrm{min}$, respectively. UHP grade hydrogen flow rate of $45 \mathrm{~mL} / \mathrm{min}$ with a corresponding air flow of $450 \mathrm{~mL} / \mathrm{min}$ were applied for FID detector. Injector and detector were set at $250^{\circ} \mathrm{C}$. Oven initial temperature was held at $210^{\circ} \mathrm{C}$ for $13 \mathrm{~min}$ and then increased to $230^{\circ} \mathrm{C}$ at the heating rate of $5^{\circ} \mathrm{C} / \mathrm{min}$ where held for $15 \mathrm{~min}$ hence, giving a total analytical time of about $27 \mathrm{~min}$. A sample volume of $0.5 \mu \mathrm{L}$ was injected using split mode as split ratio of $1: 50$.

\subsubsection{Catalyst and soap contents}

Free Fatty Acid (FFA) analysis was accomplished by titration method according to AOAC method 940.28 or AOCS Official Method Ca 5a-40. The presence of residual soap and catalyst concentration were determined by AOCS Ce 17-95 or AOCS CC 17-97 titration method using phenolphthalein as indicator to determine the catalyst concentration, and bromophenol blue to determine the soap concentration by titrating with $0.1 \mathrm{~N}$ of hydrochloric acid ( $\mathrm{HCl})$. The concentrations of residual catalyst $\left(N_{c, \text { catalyst }}\right)$ and residual soap $\left(N_{c, \text { soap }}\right)$ were normalized with initial catalyst and soap concentration in the crude biodiesel and calculated using Eq. (2) and Eq. (3), respectively.

$$
m_{\text {catalyst }}=\frac{V_{\text {acid,catalyst }} \times 0.01 \times M W_{\text {catalyst }} \times 1000}{m_{\text {sample }}}
$$

where $m_{\text {catalyst }}$ is the amount of catalyst remaining content ( $\mathrm{ppm}), m_{\text {sample }}$ is the weight of biodiesel sample (g), $V_{\text {acid,catalyst }}$ is the volume of added hydrochloric acid until solution change color from pink to colorless $(\mathrm{mL})$ and $M W_{\text {catalyst }}$ is the molecular weight of catalyst.

$$
m_{\text {soap }}=\frac{V_{\text {acid,soap }} \times 0.01 \times M W_{\text {soap }} \times 1000}{m_{\text {sample }}}
$$

where $m_{\text {soap }}$ is the amount of soap remaining content (ppm), $m_{\text {sample }}$ is the weight of biodiesel sample $(\mathrm{g})$, $V_{\text {acid,soap }}$ is the volume of added hydrochloric acid until solution change color from yellow to blue-violet $(\mathrm{mL})$ and $M W_{\text {soap }}$ is the molecular weight of soap based on oleic acid as raw material.

\section{Results and discussions}

Electrocoagulation (EC) with AC electrical current at high voltage were specifically designed for the separation of glycerol from biodiesel. Optimal conditions were determined according to the separation time, the separation efficiency, the purity of biodiesel and the remaining catalyst and soap contents.

\subsection{Electrocoagulation system design}

The characteristics of corona discharges from different types of electrodes (sharp, bar and point to point electrodes) of high voltage supply were investigated to enhance the understanding of corona discharges which using iron electrode. The electrode design was important to choose as effective factors on the separation performance. The electrode configurations shown in Fig. 3 were investigated.

\subsubsection{Effect of electrodes configuration and vertical- distance between electrodes}

Type of $\mathrm{Fe}$ electrode and distance between two electrodes can influence the removal of glycerol from biodiesel as illustrated in Fig. 4 by EC process with different distance. For EC process with applied AC electrical current at high voltage of $3 \mathrm{kV}$ for a separation time of $240 \mathrm{~s}$, the separation efficiencies for bar and point electrode types at the same level $(\mathrm{d}=0 \mathrm{~cm})$ of two electrodes were at the lowest of $94.2 \%$ and $96.0 \%$, respectively. As increased the vertical-distance between electrodes to $3 \mathrm{~cm}$, the separation efficiency was improved in comparing with the same level. However, the separation efficiency was decreased as increased the distance higher than $3 \mathrm{~cm}$. Overall, the separation efficiency was still higher than at the same level of electrodes. Therefore, the maximum performance was achieved at the distance of $3 \mathrm{~cm}$. Point-to-point electrode arrangement with the vertical-distance between electrodes of $3 \mathrm{~cm}$ could enhance the separation efficiency up to $99.8 \%$. It can be observed that electric field strength increases as gap length decreases. Thus, for smaller gap distances, ions or electrons took shorter time to reach the needle tip [29] resulting in negative charge and positive charge of electrical potential of glycerol molecule was very high under high electric stress leading to reduce repulsive force. Hence, they could get closer to form larger particles and then ready to settle or precipitate.

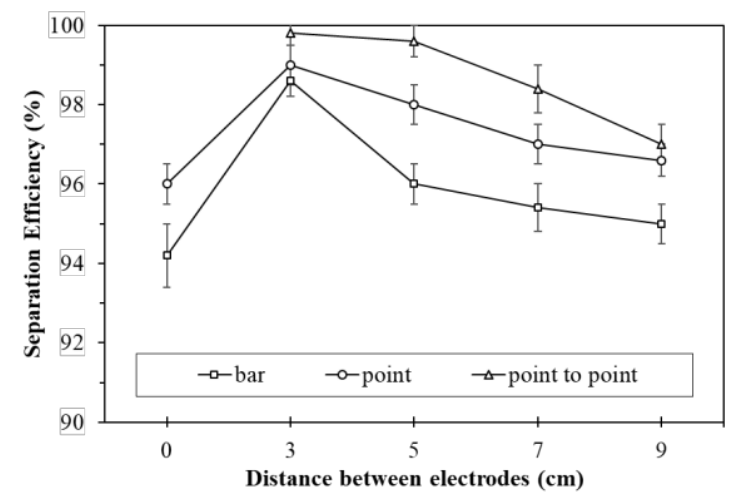

Fig. 4. Effect of Fe electrode type and vertical-distance between electrodes on separation efficiency of electrocoagulation process with applied $\mathrm{AC}$ at $3 \mathrm{kV}$ for separation time of $240 \mathrm{~s}$. 


\subsubsection{Effect of electrodes configuration and applied $A C$ at high voltage}

The separation efficiency of EC process with the vertical-distance between electrodes of $3 \mathrm{~cm}$ and for a separation time of $240 \mathrm{~s}$ was improved by increasing applied AC electrical current voltage from $1 \mathrm{kV}$ to $9 \mathrm{kV}$ as shown in Fig. 5. By applying the high voltage, the electrical field is generated around the tip of electrode, especially the sharpest point with the highest electric field strength distribution [30]. The electrical field strength is gradually weakened with distance in any direction. While, another electrode that connected to ground has almost zero electric field. Therefore, by using point-to-point electrode, the electric field magnitude was higher, and more ionizations can take place and subsequently more agglomerations of glycerol particles. As a result, the dispersion attractive-force became larger and increased the rate of aggregation and coalescence due to the raise ionization level of ions affecting the distribution of the ion charges of glycerol particles in the biodiesel and glycerol mixture.

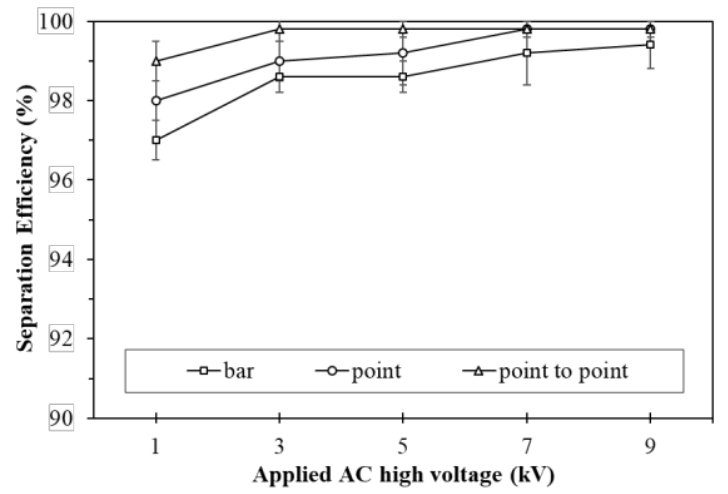

Fig. 5. Effect of Fe electrode type with vertical-distance between electrodes of $3 \mathrm{~cm}$ and applied AC electrical current at high voltage on separation efficiency of electrocoagulation process for separation time of $240 \mathrm{~s}$.

Interestingly, the separation efficiencies for point-topoint electrode configuration were not significantly improved for the voltage higher than $3 \mathrm{kV}$. However, the highest separation efficiencies of $99.8 \%$ and $99.4 \%$ with point and bar electrode types were achieved at $7 \mathrm{kV}$ and $9 \mathrm{kV}$, respectively.

\subsubsection{Effect of vertical-distance between electrodes}

Point-to-point Fe electrode type was chosen as the best design of electrode configuration for electrocoagulation separation process. Inter-electrodes distance or vertical distance (d) as shown in Fig. 3 (e) influence the electric field strength distribution and the electrical field stress between inter-electrode gap. However, the distance between inter-electrode of $1 \mathrm{~cm}$ can lead to the breakdown and spark discharge was more easily occurred. The effect of breakdown can damage the power equipment, short circuit the system, or even cause hazardous fire. For safety operation with maximum separation efficiency of $99.8 \%$, the smallest distance of inter-electrode of $3 \mathrm{~cm}$ would be practical for a separation time of $240 \mathrm{~s}$, while the distance of $2 \mathrm{~cm} \mathrm{did}$ not provide good performance for a short separation time as shown in Fig. 6. This may be explained by the fact that ions can be easily chained to travel from cathode to anode or anode to cathode such as electron pathway as result of current flow then leads to avalanche breakdown before storage of ionized molecules. The separation efficiency was influenced by a function of gap distance between both point electrodes that affected in glycerol precipitation. When the farther gap was set, it reduced the field enhancement and critical high field region to a conductive ion in mixture [31].

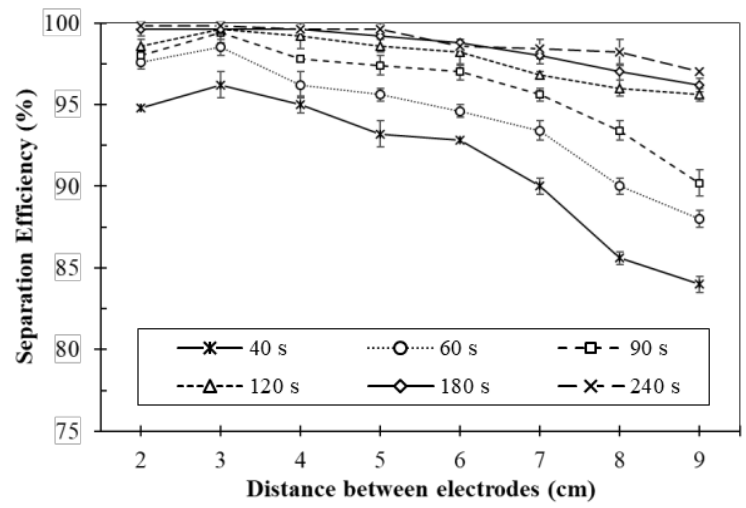

Fig. 6. Effect of vertical-distance between electrodes on separation efficiency of electrocoagulation process using $\mathrm{Fe}$ electrode with point-to-point electrode type and applying AC electrical current at high voltage of $3 \mathrm{kV}$.

\subsubsection{Effect of separation time}

By applying EC with AC electrical current at high voltage to the biodiesel and glycerol product mixture, the clear interface between the biodiesel and glycerol layers was first observed at a short separation time within 60$70 \mathrm{~s}$. However, the purity of biodiesel product was not met the standard and remaining catalyst and soap contents were still high. Therefore, the separation time was extended to $600 \mathrm{~s}$.

Fig. 7 showed the effect of separation times $(30 \mathrm{~s}$ to $600 \mathrm{~s}$ ) on the separation efficiencies varying applied AC voltage with the point-to-point electrode configuration and the distance between two electrodes of $3 \mathrm{~cm}$. The removal of glycerol was faster with higher applied AC voltage. To achieve the separation efficiency more than $99 \%$, the separation time had to be longer than $120 \mathrm{~s}$ for the applied AC voltage less than $9 \mathrm{kV}$. The optimal condition for electrocoagulation separation process with the maximum separation efficiency of $99.8 \%$ were determined at the shortest separation time with low energy consumption (low applied AC voltage) and it was found to be at $3 \mathrm{kV}$ for $240 \mathrm{~s}$ with the point-to-point Fe electrode configuration. Although the separation efficiency was increased with the separation time, but after $420 \mathrm{~s}$ the efficiency was not significant for all applied AC voltages possibly due to the destabilizing effect at low particle concentrations of glycerol in the biodiesel-rich phase as visually clear in the upper phase. 


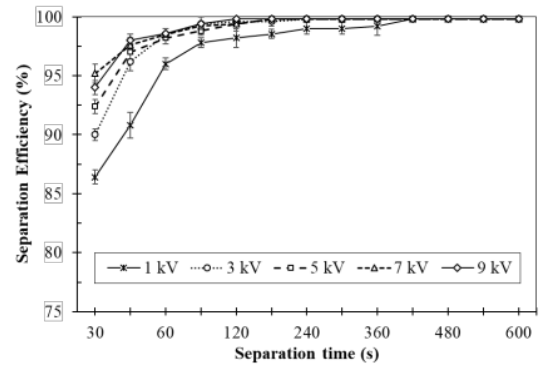

Fig. 7. Effect of separation time on separation efficiency of electrocoagulation process using Fe electrode with point-topoint electrode type with vertical-distance between electrodes of $3 \mathrm{~cm}$ varying applied $\mathrm{AC}$ electrical current at high voltage.

\subsection{Separation efficiency before purification process}

After the reaction was completed, the product mixture could settle, and two layers were appeared. In the upper biodiesel-rich phase or methyl ester-rich phase, minor residual soap and free glycerol remained suspending in this phase along with small amount of unreacted monoglycerides, diglyceride and triglyceride. While the lower glycerol-rich phase contained largely some of excess methanol and most of catalyst and soap. Moreover, these residuals could suspend and distribute between these two phases, especially unreacted alcohol, residual catalyst and trace glycerol.

\subsubsection{Remaining soap and catalyst contents}

The performance of electrocoagulation separation process was investigated by evaluating the quantity of flocculation agents in the biodiesel final product. Fig. 8 showed the depletion of catalyst was largely removed by EC process for a separation time longer than $240 \mathrm{~s}$. Although after applying EC for $30 \mathrm{~s}$, the clear interface between the biodiesel and glycerol layers without cloudy solution in the upper biodiesel-rich phase was firstly observed and presumably most of catalyst was precipitated and dissolved in the glycerol-rich phase, but normalized remaining catalyst and soap contents were still high until the separation time of $180 \mathrm{~s}$ when the contents were significantly reduced. Almost $90 \%$ of catalyst dissolved into the glycerol-rich phase at $180 \mathrm{~s}$ which was in the agreement with the gravitational settling separation at $>24 \mathrm{~h}(90-94 \%)$ [32]. Furthermore, the amount of methanol remaining in biodiesel phase could be caused strong electrolyte in biodiesel phase although the polar nature of its compound was preferably dissolved in glycerol phase [33].

Nevertheless, some catalyst, moisture and unreacted methanol in the upper layer of biodiesel-rich phase cannot be easily eliminated and may interrupt the reaction by causing a thermodynamics equilibrium will not be reached with the mixing and the reaction becomes limited by mass transfer of the two phases due to reversible reaction. Another reason is that alkali catalyst can generate small quantities of soap as emulsifying from free fatty acid presented at least in small qualities based on oil raw materials. The concentration value catalyst remaining in the biodiesel layer at $30 \mathrm{~s}$ was 0.367 and it reduced to 0.093 at the separation time of $180 \mathrm{~s}$. Thus, the required separation time to remove all impurities, especially catalyst, was longer than $240 \mathrm{~s}$ with the use of EC.

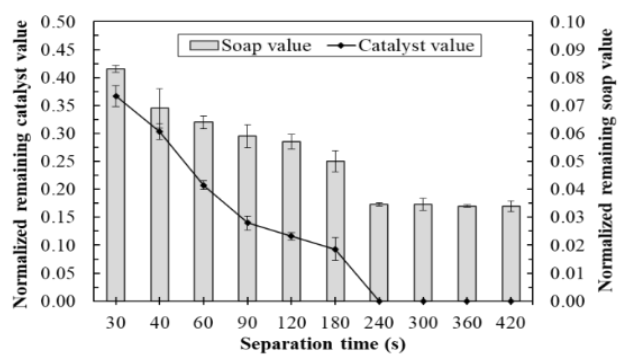

Fig. 8. Normalized concentrations of remaining catalyst and soap from the electrocoagulation process using $\mathrm{Fe}$ point-topoint electrodes with vertical-distance between electrodes of 3 $\mathrm{cm}$ and applied AC electrical current at high voltage of $3 \mathrm{kV}$.

However, soap remaining could not be easily removed as increasing in the separation time using $\mathrm{EC}$ process since the transesterification reaction occurs in parallel with saponification reactions with accompanied by catalyst consumption. Still, the normalized concentration of soap content was significantly reduced from 0.083 to 0.035 within $240 \mathrm{~s}$. After separation time for $240 \mathrm{~s}$, it did not change and remain at the same value of 0.034 because mostly soap was entrapping in the glycerol phase and removed from biodiesel layer due to a strong affinity with glycerol under high electric field.

\subsubsection{Methyl ester content}

The conversion of methyl ester was increased from $78.9 \%$ to $95.2 \%$ from the separation time of $30 \mathrm{~s}$ to $240 \mathrm{~s}$ using electrocoagulation separation process while the normalized concentrations soap decreased from 0.083 to 0.034 (Fig. 9). By increasing the separation time, most of the soap was precipitated in glycerol phase resulting in decreasing in catalyst and soap content. However, after $240 \mathrm{~s}$, normalized remaining soap content was not significantly changed. Therefore, it is necessary to further purify with water washing to complete the reaction with excess of reagents via reversible transesterification.

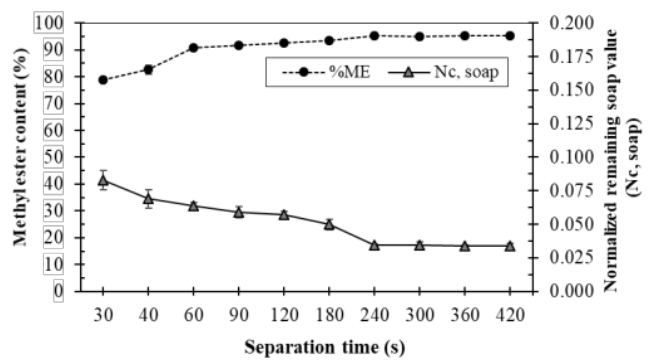

Fig. 9. Normalized methyl ester content and normalized concentration of soap in the biodiesel final product from the electrocoagulation process using $\mathrm{Fe}$ point-to-point electrodes with vertical-distance between electrodes of $3 \mathrm{~cm}$ and applied $\mathrm{AC}$ electrical current at high voltage of $3 \mathrm{kV}$. 


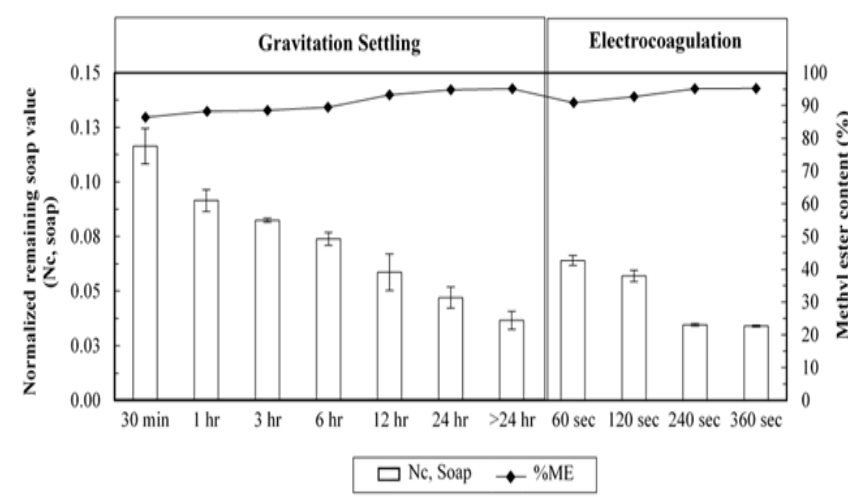

Fig. 10. Comparison of separation performance between gravitation settling in a glass cylindrical chamber and electrocoagulation process using Fe electrode with point-topoint electrode type and vertical-distance between electrodes of $3 \mathrm{~cm}$ and applying AC electrical current at high voltage of $3 \mathrm{kV}$.

Table 2. Characteristics of biodiesel final product.

\begin{tabular}{|l|l|l|c|c|c|}
\hline \multirow{2}{*}{ Parameters } & \multirow{2}{*}{ Units } & \multirow{2}{*}{ Method } & $\begin{array}{l}\text { LSTM } \\
\text { D6751 }\end{array}$ & EN14214 & \multirow{2}{*}{ Results } \\
\hline Ester content & $\%$ & EN14103 & $-*$ & $\geq 96.5$ & $\begin{array}{c}98.89 \pm 0.2 \\
5\end{array}$ \\
\hline Acid value & $\begin{array}{l}\mathrm{mg} \\
\mathrm{KOH} / \mathrm{g} \\
\text { of sample }\end{array}$ & ASTM D664 & $-*$ & $0.50 \mathrm{max}$ & 0.043 \\
\hline $\begin{array}{l}\text { Iodine value } \\
\text { (Wijs) }\end{array}$ & $\begin{array}{l}\mathrm{g} \text { iodine/ } \\
100 \mathrm{~g}\end{array}$ & EN14111-03 & $-*$ & $120 \mathrm{max}$ & 53.0 \\
\hline $\begin{array}{l}\text { Kinematic } \\
\text { Viscosity; } \\
40^{\circ} \mathrm{C}\end{array}$ & $\mathrm{mm}^{2} / \mathrm{s}$ & $\begin{array}{l}\text { ASTM } \\
\text { D445-17a }\end{array}$ & $\begin{array}{c}1.9 \text { to } \\
6.0\end{array}$ & $3.5-5.0$ & 4.781 \\
\hline $\begin{array}{l}\text { Pour point } \\
{ }^{\circ} \mathrm{C}\end{array}$ & $\begin{array}{l}\text { ASTM } \\
\text { D5950-14 }\end{array}$ & $-*$ & $-* * *$ & 11.0 \\
\hline Cloud point & ${ }^{\circ} \mathrm{C}$ & $\begin{array}{l}\text { ASTM } \\
\text { D5771-17 }\end{array}$ & $-* *$ & $-* * *$ & 12.0 \\
\hline Flash point & ${ }^{\circ} \mathrm{C}$ & $\begin{array}{l}\text { ASTM D98- } \\
16 \mathrm{a}\end{array}$ & $130 \mathrm{~min}$ & $101 \mathrm{~min}$ & 175.0 \\
\hline
\end{tabular}

Note: * Not specified

** The cloud point of biodiesel is generally higher than that of petroleum-based diesel fuel and should be taken into consideration when blending.

*** Not specified. EN14214 uses time and location dependent values for the cold filter plugging point (CEPP) instead.

\subsection{Comparison between gravitational settling and electrocoagulation separation processes}

A layer of glycerol and methyl esters started to be visibly clear at the interface for about $300 \mathrm{~s}$ of gravitational settling (GS) while mostly complete settling takes place between $2 \mathrm{~h}$ to $24 \mathrm{~h}$ or more process time. The experiments were carried out of excess methanol/oil molar ratio of $6: 1$, sodium hydroxide derivative-catalyst, constant stirring speed of $480 \mathrm{rpm}$ at $65^{\circ} \mathrm{C}$ for $1 \mathrm{~h}$. Fig. 10 showed the effect of variation of GS separation time from $5 \mathrm{~min}$ to more than $24 \mathrm{~h}$. Separation by GS required greater force and more time to separate glycerol from biodiesel comparing with shorten time of EC separation process.

Moreover, it was still found that normalized concentration of remaining catalyst and soap contents in biodiesel layer were ranging from 1.42 to 0.11 and 0.158 to 0.037 , respectively, while EC separation process after
240 s separation time did not find any of residual catalyst. In this work, the assisted post-treatment before washing step by electrocoagulation separation process was considered as an effective and economizing method.

The remaining catalyst and soap content could be removed with further purification with water-washing. As the water to biodiesel increased, the amount of the residues and contaminant in the final product decreased. However, biodiesel product could be lost with many times of washing that can be lost of biodiesel product. For this research, only two water-washing was required to improve the quality of biodiesel and successfully within the standard specifications for biodiesel.

\section{Conclusion}

This research investigated the electrocoagulation technology as an alternative method for separation of biodiesel from glycerol by applying alternating current (AC) at high voltage and low amperage. To achieve the separation efficiency of $99.8 \%$, electrocoagulation separation process was operated the optimal condition of applied AC electrical current at high voltage of $3 \mathrm{kV}$ with $\mathrm{Fe}$ point-to-point electrode and the inter-electrodes distance of $3 \mathrm{~cm}$. After $40 \mathrm{~s}$ of separation time, the clear interphase of biodiesel-rich and glycerol-rich layers were observed. The separation was completed at $240 \mathrm{~s}$ and after only 3 times of water-washing unreacted catalyst was eliminated and amount of soap was reduced. Biodiesel final product was within the biodiesel standards of EN 14214 and ASTM D6751.

The use of AC electrical current at high voltage $(\mathrm{kV})$ for separation biodiesel from glycerol provides a better separation efficiency for post-treatment followed by purification process with shorten in the separation time, the catalyst remaining and reduce soap content eliminate and also reduce number of water-washing times for the purification. The application of electrocoagulation can merge molecule of glycerol as well as other charged residues into larger particulates under high stress electric field between tip electrode of point to point electrode. This effect can adhere molecule glycerol and unreacted molecule together and produce aggregates of larger size, which can settle out faster than gravitational settling or conventional sedimentation. Furthermore, the successful of this separation technique has various parameters such as different types electrode material and energy consumption will require to investigate in future work.

Electrocoagulation separation process can be the practical configuration in order to retrofit the existing process at the industrial plant and a new feasible application for electrocoagulation in the separating another mixture component or multicomponent mixture. However, due to the high capital cost and safety in the construction of the high voltage power system, it is not commercially attractive. Therefore, the investigation on the electrocoagulation with $\mathrm{AC}$ electrical current at low voltage up to $100 \mathrm{~V}$ was explored in the next research.

The authors would like to thank King Mongkut's University of Technology North Bangkok (Research 
Grant Contract No. KMUTNB-GOV-58-17) for the financial support.

\section{References}

1. A.S. Peiter, P.V.S. Lins, L. Meili, J.I. Soletti, S.H. V. Carvalho, W.R.O. Pimentel, S.M.P. Meneghetti, J King Saud Univ Sci. (2018)

2. E.N. Ali, C.I. Tay, Procedia Eng. 53, 7-12. (2013)

3. N. Banerjee, R. Ramakrishnan, T. Jash, Energy Procedia. 54, 161-165 (2014)

4. M.A. Ahmad Farid, M.A. Hassan, Y.H. Taufiq-Yap, Y. Shirai, M.Y. Hasan, M.R. Zakaria, J Clean Prod. 165, 262-272. (2017)

5. M. Ilmi, A. Kloekhorst, J. Winkelman, G. Euverink, C. Hidayat, H. Heeres, Chem Eng J. 321, 76-85 (2017)

6. G. Kraai, B. Schuur, F. Van Zwol, H. Van de Bovenkamp, H. Heeres, Chem Eng J. 154(1-3), 384389. (2009)

7. K. Kara, F. Ouanji, E.M. Lotfi, M.E. Mahi, M. Kacimi, M. Ziyad, Egypt J Pet. 27(2), 249-255 (2018)

8. P. Verma, M.P. Sharma, G. Dwivedi, Egypt J Pet. 25(1), 91-95 (2016)

9. A. Nouredin, M.M Shirazi, J. Tofeily, P. Kazemi, E. Motaee, A. Kargari, M. Tabatabaei, Sep Puri Technol. 132, 272-280 (2014)

10. A. Abeynaike, A. Sederman, Y. Khan, M. Johns, J. Davidson, M. Mackle, Chem Eng Sci. 79, 125-137 (2012)

11. J. Saleh, A.Y. Tremblay, M.A. Dubé, Fuel. 89(9), 2260-2266. (2010)

12. J. Ye, Y. Sha, Y. Zhang, Y. Yuan, H. Wu, Bioresour Technol. 102(7), 4759-4765 (2011)

13. B. Johnson, B. Melde, M. Moore, A. Malanoski, J. Taft, Materials. 10(6), 682. (2017)

14. I. Zongo, J.P. Leclerc, H.A. Maïga, J. Wéthé, F. Lapicque, Sep Puri Technol. 66(1), 159-166 (2009)

15. C.T. Wang, W.L. Chou, Y.M. Kuo, J Hazard Mater. 164(1), 81-86 (2009)

16. S. Zhang, H. Zheng, X. Tang, Y. Sun, Y. Wu, X. Zheng, Q. Sun, J Taiwan Inst Chem Eng, (2018)

17. S.Y. Choy, K.M.N. Prasad, T.Y.Wu, R.N. Ramanan, Int J Environ Sci Technol. 12(1), 367-390 (2013)
18. M.G.H. Nampoothiri, A.M. Manilal, P.A. Soloman, Proc Technol. 24, 603-610 (2016)

19. R. Kamaraj, P. Ganesan, J. Lakshmi, S. Vasudevan, Environ Sci Pollut R. 20(1), 399-412 (2012)

20. K. Vuthivat, T. Kangsadan, S. Chalermwisutkul. International Conference Proceeding of PACCON 2012, (2012)

21. W. Garnwitayee, T. Kangsadan, S. Chalermwisutkul, ICBEE 2012 (2012)

22. P. Wanking, A.F. Muhtadin, T. Kangsadan, International Conference Proceeding of PACCON 2019 (2019)

23. M. Obpirompu1, R. Amprirojanawong, T. Kangsadan, International Conference Proceeding of PACCON 2019, (2019)

24. BS EN14103:2011. Fat and oil derivatives - Fatty Acid Methyl Esters (FAME)-Determination of ester and linolenic acid methyl ester contents. BSI. London, Great Britain (2011)

25. Haus, A. Hermann, J.R. Melcher, Electromagnetic Fields and Energy. Englewood Cliffs, NJ: PrenticeHall (1989). ISBN: 9780132490207.

26. A. Edgar, C. Coronel, Rev. Bol. Quim. 28, 41-45 (2011)

27. A. Rajab, A. Sulaeman, S. Sudirham, S. Harjo, ITB Journal of Engineering Science 43, 191-208 (2011).

28. Krohne group. URL:https://krohne.com/en/services/ dielectric-constants/ (2019)

29. N. Baruah, M. Maharana, S.K. Nayak, 2nd International Conference on Power, Energy and Environment (ICEPE): Towards Smart Technology, (2018)

30. G. Altamimi, H.A. Illias, N. Mokhtar, H. Mokhlis, A.H.A. Bakar, IEEE International Conference on Power and Energy (PECon) (2014)

31. A.L. Kupershtokh, E.I. Palchikov, D.I. Karpov, I. Vitellas, D.P. Agoris, V.P. Charalambakos, J Phys D: Applied Physics. 35(23), 3106-3121 (2002)

32. W. Zhou \& D. G. B. Boocock, J Am Oil' Chem Soc. 83(12), 1041-1045 (2006)

33. A. Gonzalo, M. García, J. Luis Sánchez, J. Arauzo, J.A. Peña, Ind Eng Chem Res. 49(9), 4436-4443 (2010) 